\title{
Should we take simulation out of the lab?
}

\author{
Jason Bryant*1 \\ ${ }^{1}$ Pediatric Anesthesiologist, Nationwide Children's Hospital, Columbus, OH 43205, USA
}

Education is an important part of the practice of medicine. The Hippocratic oath comments on education in medicine stating "teach them this art, if they shall wish to learn it, without fee or contract; and that by the set rules, lectures, and every other mode of instruction" [1]. One of the skills that every anesthesia resident must become an expert in prior to graduation is fiberoptic intubation. Fiberoptic intubation is key for safely securing the difficult airway. If this skill set lacking harm to patients will certainly occur. A problem with teaching a lifesaving skill in situations where a skilled practitioner is needed to prevent patient harm is that an unskilled novice may cause harm to the patient while they are learning. This is one reason that simulation is often used to teach fiberoptic intubations (Figures 1, 2 and 3).

Most simulation training takes place outside clinical duties. Residents at many institutions are involved in simulation training at a simulation center that has been designed to reflect the operating room environment. Fiberoptic intubation can be taught in this setting has had differing outcomes in recent studies. These results have varied from having extreme standard deviations in skills when learning nasal endoscopy on a fiberoptic simulation and using a simulator could advance resident skills to levels similar to that of an attending physician $[2,3]$. One of the major limitations and benefits of simulation education is that it removes the learner from real events. By reducing the risk of harm it allows for high stakes decisions and skills to be performed solely by the learner. However, it also removes the learner from the flesh and blood of real patient interactions. The best fiberoptic simulator still lacks the stereotactic feel and complexity of an actual patient.

The question is not whether simulation is beneficial in training but how to utilize it in the most optimal manner. A proposal has been made to utilize simulation in the operating room environment for fiberoptic intubation [4]. Simulation in the operating room would allow for repetition in a safe low risk environment prior to performance with an actual patient. As the learner gained skills immediately prior to the patient interaction the loss of skills as can be seen between long

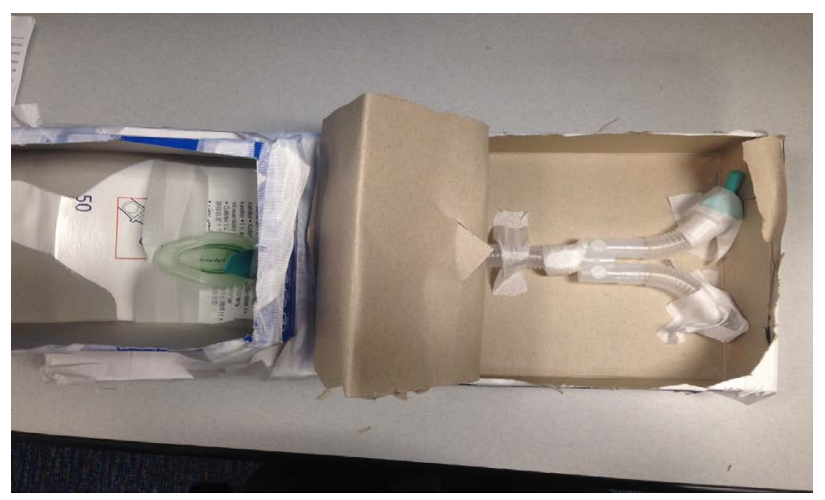

Figure 1. Fiberoptic tube. time periods could be minimized and the skill itself reinforced. To perform the fiberoptic intubation safely, calmly, and with confidence,

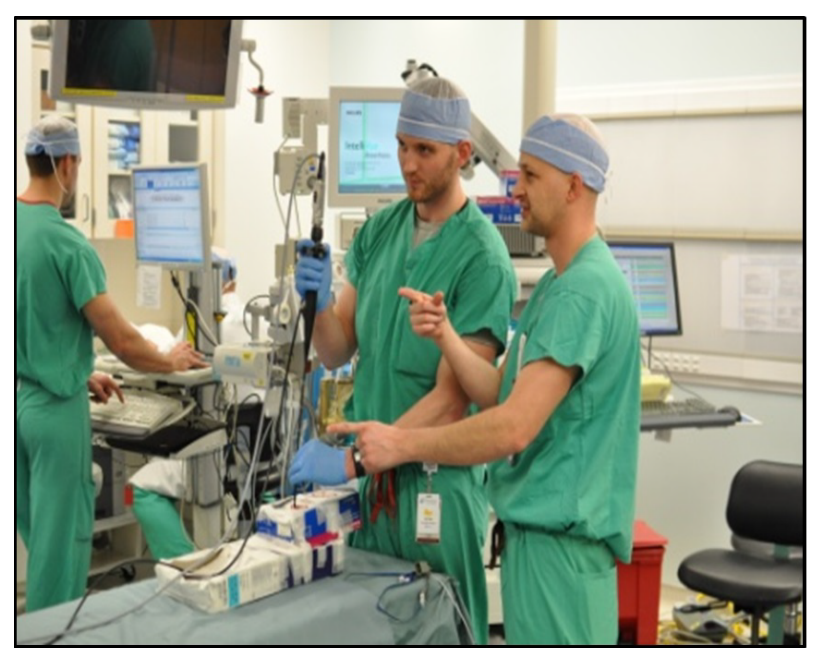

Figure 2.The learner practices with the simulator in the actual operating room where the fiberoptic intubation is to take place.

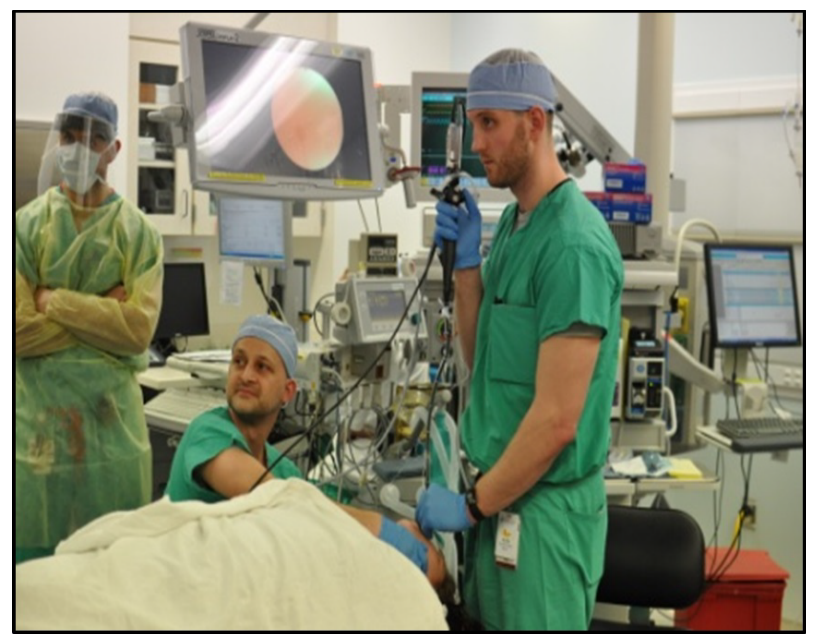

Figure 3. Then the patient is intubated in the same environment directly after the simulator training if performed.

Correspondence to: Jason Bryant, MD, Pediatric Anesthesiologist, Nationwide Children’s Hospital, Columbus, OH 43205, USA, E-mail: Jason.Bryant@ nationwidechildrens.org

Received: February 28, 2017; Accepted: March 13, 2017; Published: March 15, 2017 
it was elected to recreate the airway and perform a simulation prior to intubation. The airway was simulated using common tubing from the OR of approximate size, shape, and angles of pediatric larynx, trachea, and bronchi. The passage contained landmarks marked with different colors to serve as checkpoints.

Fiberoptic intubation is not the only skill that could be taught in this manner. Invasive procedures such as nerve blocks, epidural catheters and arterial lines all could be reviewed directly prior to performance. Several commercial simulators are available but simple devices can be fashioned to review without high expense. Simulation in the operating room directly prior to patient interaction would benefit the teacher, the learner and ultimately the patient. The learner would benefit from the review. The teacher would benefit by assessing the learners skill set prior to patient contact. Therefore, addressing the learner's gaps in knowledge or deciding that the learner is not yet prepared to perform the skill on an actual patient. The patient would benefit from all of these reasons while still being an important part the education process.

\section{References}

1. Collier PF, Son (1910)Oath of hippocrates. Harvard Classics 38.

2. Goldmann K, Steinfeldt T (2006) Acquisition of basic fiberoptic intubation skills with a virtual reality airway simulator. J Clin Anesth18: 173-178. [Crossref]

3. Dalal PG, Dalal GB, Pott L, Bezinover D, Prozesky J, Bosseau Murray W (2011) Learning curves of novice anesthesiology residents performing simulated fiberoptic upper airway endoscopy. Can J Anaesth 58: 802-809. [Crossref]

4. Bryant J, Schwab C, Powell M (2014) Squeezing teaching strategies into clinical practice. Utilizing simulation in the operating room environment for fiberoptic intubation. Poster session presented at the Annual Society of Pediatric Anesthesia meeting.

Copyright: (C2017 Bryant J. This is an open-access article distributed under the terms of the Creative Commons Attribution License, which permits unrestricted use, distribution, and reproduction in any medium, provided the original author and source are credited. 